BULl. AUSTRAL. MATH. SOC.

VOL. 36 (1987) 367-374

\title{
EACH WEAKLY COUNTABLY DETERMINED ASPLUND SPACE ADMITS A FRÉCHET DIFFERENTIABLE NORM
}

\author{
M. FABIAN
}

If an Asplund space is weakly countably determined, then it admits an equivalent Fréchet differentiable norm and is weakly compactly generated. If on an Asplund space there exists an equivalent Gâteaux differentiable norm, then its dual has a projectional resolution of identity.

All Banach spaces considered in this note are real. The explanation of the notions not introduced here can be found in [3], [4], [11] and [14].

There exists an open problem, over twelve years old, as to whether every Asplud space admits an equivalent norm with some smoothness property. It is an old and well known fact that every separable Asplund space can be equivalently renormed in such a way that this new norm is Frechet differentiable and the dual norm is locally uniformly rotund; the historical references to this result can be found in [10] and [3, pp. 113-119, 125]. John and zizler [10] extended this theorem to all weakly compactly generated Banach spaces which possess a continuously Fréchet differentiable real valued function with bounded nonempty support. We recall that such spaces are Asplund, as, for instance, Ekeland and Lebourg have shown. In this note we weaken the assumptions of the theorem

Received 29 October 1986. The author would like to thank Dr. Kamil John for discussions on the subject. Thanks are also due to Dr. W. Marciszewski for drawing our attention to the paper [11].

Copyright Clearance Centre, Inc. Serial-fee code: 0004-9729/87 $\$ A 2.00+0.00$. 
of John and zizler as follows.

THEOREM 1. Let $(X,\|\cdot\|)$ be a weakly countably determined Asplund space.

Then $X$ admits an equivalent Frêchet differentiable norm whose dual norm is locally uniformly rotund. Moreover $X$ is weakly compactly generated.

At first glance the above theorem supports the confecture that every Asplund space has an equivalent Frechet differentiable norm. However, we should mention that the spaces whose duals possess equivalent dual locally uniformly rotund norms form a proper subclass of the class of spaces admitting an equivalent Fréchet differentiable norm. In fact, Talagrand [13] has shown that the space $C([0,8])$ has an equivalent Fréchet differentiable norm, yet no dual norm is even strictly convex

All that is needed for the proof of Theorem 1, but one step, can be found in a paper of the author [5], where (among other things) ideas of John and zizler [9], [10] are extended a little. This gap can be filled up by a Mercourakis's extension of a renorming theorem of Amir and Lindenstrauss [11, Theorem 4.6], together with a special case of a striking result of Jayne and Rogers [6, Theorem 8] saying that a norm-weak* continuous mapping from an Asplund space into its dual is a point wise limit of a sequence of norm-norm continuous mappings. However we feel that the proof of Theorem 1 should be given in more detail here.

Proof. By [11, Theorem 4.6] the dual $X^{*}$ admits an equivalent dual strictly convex norm. Hence $X$ admits an equivalent Gâteaux differentiable norm $|\cdot|$, say. Let $D$ denote the Gateaux derivative of $|\cdot|^{2}$. Then $D$ is a norm-weak* continuous mapping from $X$ into $X^{*}$. As $X$ is Asplund, $X^{*}$ is weak* dentable. Thus, according to [6, Theorem 8], there exists a sequence $\left\{D_{n}\right\}$ of norm-norm continuous mappings from $X$ into $X^{*}$ such that $D x$ lies in the norm closure of the set $\left\{D_{1} x, D_{2} x, \ldots\right\}$ for each $x \in X$. Further we shall need the following lemma.

LEMMA. Let the assumptions of Theorem 1 hold and let $\mu$ denote the first ordinal of cardinality dens $X$. 
Then on $X$ there exists $a$ "Zong sequence" $\left\{P_{\alpha}: \omega \leq \alpha \leq \mu\right\}$ of linear projections such that $P_{\mu}$ is the identity, and for all $\omega \leq \alpha \leq \mu$ the following relations hold

$\left\|P_{\alpha}\right\|=1, P_{\alpha \beta} P_{\beta}=P_{\beta} P_{\alpha}=P_{\beta}$ if $\beta \leq \alpha$, dens $P_{\alpha} X \leq \overline{\bar{\alpha}}$, $\underset{\beta<\alpha}{\cup} P_{\beta+1} X$ is dense in $P_{\alpha} X$ if $\omega<\alpha$, and $P_{\alpha}^{*} X^{*}>\underset{\beta<\alpha}{\cup} \underset{n<\omega}{\cup} D_{n}\left(P_{e} X\right)$.

Proof. We can proceed as in the proof of [14, Theorem 1] with some small changes in Step VI.

Having proved the Lemma we shall show that the adjoint projections $P_{\alpha}^{*}$ enjoy the same properties as $P_{\alpha}$ do save the last one. Clearly $P_{\mu}^{*}$ is the identity, $\left\|P_{\alpha}{ }^{*}\right\|=1$, and $P_{\alpha}{ }^{*} P_{\beta}^{*}=P_{\beta}^{*} P_{\alpha}^{*}=P_{\beta}^{*}$ if $\beta \leq \alpha$. Further we claim that the restriction mapping $f \rightarrow f / P_{\alpha} X$ maps $P_{\alpha}^{*} X^{*}$ onto $\left(P_{\alpha} X\right)^{*}$ isometrically. Indeed, fix $\omega \leq \alpha \leq \mu, f \in P_{\alpha}^{*} X^{*}$, and let $\varepsilon>0$ be arbitrary. We can take $x \in X,\|x\|=1$; such that

$\|f\|-\varepsilon<f(x)$. But

$$
f(x)=P_{\alpha}^{*} f(x)=f\left(P_{\alpha} x\right) \leq\left\|f /_{P_{\alpha}}\right\|\left\|P_{\alpha} x\right\| \leq\left\|f /_{P_{\alpha}}\right\| .
$$

Hence $\|f\|-\varepsilon<\left\|f / P_{P^{X}}\right\|$, and letting $\varepsilon$ go to 0 , we get that $\|f\|=\left\|f /_{P_{\alpha}}\right\|$. Also, if $g \in\left(P_{\alpha} X\right)^{*}$ and $\bar{g} \in X^{*}$ is such that $\bar{g} / P_{\alpha} X=g$, then for all $x \in P_{\alpha} X$ we have $P_{\alpha}{ }^{*} \bar{g}(x)=\bar{g}\left(P_{\alpha} x\right)=g(x)$. Thus the restriction mapping is a surjective isometry. Now, as $X$ is Asplund, so is $i$ ts subspace $P_{\alpha} X$, and consequently, dens $P_{\alpha}^{*} X^{*}=$ dens $\left(P_{\alpha} X\right)^{*}=$ dens $P_{\alpha} X \leq \overline{\bar{\alpha}}$.

It remains to prove that for all limit ordinals $\omega<\alpha \leq \mu \underset{B<\alpha}{\cup} P_{B}{ }^{*} X^{*}$ is norm dense in $P_{\alpha}^{*} X^{*}$. Fix such an $\alpha$. We take $f \in P_{\alpha}^{*} X^{*}$ and let $\varepsilon>0$ be arbitrary. By the Bishop Phelps theorem we know that the set $\left\{D x / P_{\alpha} X: \quad x \in P_{\alpha} X\right\}$ is dense in $\left(P_{\alpha} X\right)^{*}$. Hence, there is $x \in P_{\alpha} X$ such that

$$
\| f /_{P_{\alpha} X}-D x / P_{\alpha} X \mid<\varepsilon / 3
$$


Further, recalling the relation between $D$ and $\left\{D_{n}\right\}$, we can find $n$ such that

$$
\left\|D x-D_{n} x\right\|<\varepsilon / 3 .
$$

Also, since $D_{n}$ is continuous and the set $\underset{\beta<\alpha}{\cup} P_{\beta} X$ is dense in $P_{\alpha} X$, there is $\omega<\gamma<\alpha$ and $u \in P_{\gamma} X$ such that

$$
\left\|D_{n} x-D_{n} u\right\|<\varepsilon / 3 \text {. }
$$

Thus

$$
\left\|f-D_{n} u\right\|=\left\|f / P_{\alpha} X-D_{n} u / P_{\alpha}\right\|<\varepsilon / 3+\varepsilon / 3+\varepsilon / 3=\varepsilon .
$$

Now, as $u \in P_{\gamma} X$ and $\gamma+1<\alpha$, we have, by the Lemma, that $D_{n} u \in P_{\gamma+1}^{*} X^{*}$. It follows that the distance of $f$ from $\underset{\beta<\alpha}{\cup} P_{B}^{*} X^{*}$ is at most $\varepsilon$. Now letting $\varepsilon$ go to 0 we get that $f$ is in the closure of $\cup P_{\beta}^{*} X^{*}$ and so the density is proved.

Moreover, it is well known [1] and easy to prove that for each $f \in X^{*}$ and each $\varepsilon>0$ the set $\left\{\omega \leq \alpha<\mu:\left\|P_{\alpha+1}^{*} f-P_{\alpha}^{*} f\right\|>\varepsilon\right\}$ is finite.

The rest of the proof of the first part of the conclusion of Theorem 1 can be completed exactly as in [9], or, more directly, using a dual version of a renorming result of zizler [15].

THEOREM (21zler). Let $X$ be a Banach space admitting a fomily $\left\{T_{\gamma}\right\}_{\gamma \in \Gamma}$ of linear continuous mappings on $X$ such that

(i) for each $f \in X^{*}$ and each $\varepsilon>0$ the set $\left\{\gamma \in \Gamma:\left\|T_{\gamma}{ }^{*} f\right\|>\varepsilon\right\}$ is finite,

(ii) every $f \in X^{*}$ lies in the closed linear span of the set $\left\{T_{\gamma}^{*} f: \gamma \in \Gamma\right\}$,

(iii) for each $\gamma \in \Gamma$ the subspace $T_{\gamma}^{*} X^{*}$ admits an equivalent locally uniformly rotund norm $\|\cdot\|_{\gamma}$ such that $\|f\|_{\gamma} \leq i \operatorname{im} i_{\tau}$ inf $\left\|f_{\tau}\right\|_{\gamma}$ whenever $f, f_{\tau} \in T_{\gamma}^{*} X^{*}$ and $f_{\tau} \rightarrow f$ weakly*.

Then $X$ admits an equivalent Fréchet differentiable norm such that the dual norm is locally uniformly rotund. 
Proof. The construction from [15] yields on $X^{*}$ an equivalent locally uniformly rotund norm. And it is easy to verify that in our situation this new norm is weakly * lower semicontinuous, that is it is a dual norm.

Now all is prepared for completing the proof of the first part of the conclusion of Theorem 1 . We shall proceed by transfinite induction over dens $X$. If dens $X=\kappa_{0}$, we already know that the promised renorming exists [3, p. 118]. So let $N>\kappa_{O}$ be given and let us assume that the renorming exists whenever dens $X<N$. Let now $X$ be such that dens $X=N$. Then, putting $\Gamma=\{0\} \cup\{\alpha: \omega \leq \alpha<\mu\}, T_{0}=P_{\omega}$, $T_{\alpha}=P_{\alpha+1}-P_{\alpha}, \omega \leq \alpha<\mu$, the conditions (i) and (ii) in the theorem of zizlex are clearly satisfied. We shall verify (iii). We fix $\omega \leq \alpha<\mu$. Then dens $P_{\alpha+1} X \leq \overline{\bar{\alpha}}<\overline{\bar{\mu}}=\aleph$. So, by the induction hypothesis, $\left(P_{\alpha+1} X\right)^{*}$ admits an equivalent dual locally uniformly rotund norm $|\cdot|_{\alpha}$, say. Put

$$
\|f\|_{\alpha}=\left|f / P_{\alpha+1} X\right|_{\alpha}, f \in T_{\alpha}^{*} X^{*}
$$

Since $P_{\alpha+1} X^{*}$ is isometrical with $\left(P_{\alpha+1} X\right)^{*}$, and $T_{\alpha}^{*} X^{*} \subset P_{\alpha+1} X^{*}$, it follows that $\|\cdot\|_{\alpha}$ is an equivalent norm on $T_{\alpha}^{*} X^{*}$. clearly, $\|\cdot\|_{\alpha}$ is locally uniformly rotund since $|\cdot|_{\alpha}$ is. Also, if $f, f_{\tau} \in{ }^{m}{ }_{\alpha}^{*} X^{*}$ and $f_{\tau} \rightarrow f$ weakly* in $X^{*}$, then $f_{\tau / P_{\alpha+1}} \longrightarrow f / P_{\alpha+1^{X}}$ weakly* in $\left(P_{\alpha+1} X\right)^{*}$. Thus $\|f\|_{\alpha} \leq \lim \tau_{\tau}$ inf $\|\left. f_{\tau}\right|_{\alpha}$ and (iii) is verified for $\gamma=\alpha$, $\omega \leq \alpha<\mu$. For $\gamma=0$ we can proceed analogously. Therefore the theorem of zizler applies and so $X^{*}$ admits an equivalent dual locally uniformly rotund norm. The induction step is thus finished.

It remains to show that $X$ is weakly compactly generated. Let us recall that every subspace $Z$ of $X$ is weakly countably determined and Asplund, too. From the first part of the proof we then know that each such $Z$ admits projections with the same properties as the $P_{\alpha}$ have. Thus by [14, Theorem 2, I $\Rightarrow$ IV], $X$ has a shrinking Markuševic basis. So, by Troyanski, $X$ is weakly compactly generated, see [10]. 
We conclude this note by weakening the assumptions of the main theorem of [5]. Namely, regarding the quoted consequence of the theorem of Jayne and Rogers, we get

THEOREM 2. Let $X$ be an Asplund space admitting an equivalent Gâtecaux differentiable norm, or, at least, a continuous Gâtecaux. differentiable real valued function with bounded nonempty support and norm-weak* continuous derivative. Let $\mu$ denote the first ordinal of cordinatity dens $X$.

Then $X^{*}$ possesses a "Zong sequence" $\left\{Q_{\alpha}: \omega \leq \alpha \leq \mu\right\}$ of Zinear projections such that $Q_{\mu}$ is the identity, and for all $\omega \leq \alpha \leq \mu$ we have $\left\|Q_{\alpha}\right\|=1, Q_{\alpha} Q_{\beta}=Q_{\beta} Q_{\alpha}=Q_{\beta}$ if $\beta \leq \alpha$, dens $Q_{\alpha} X^{*} \leq \overline{\bar{\alpha}}$, and $\underset{\beta<\alpha}{\cup Q_{B+1} X^{*}}$ is dense in $Q_{\alpha} X^{*}$ if $\omega<\alpha$.

However, these projections cannot in general be made weak*-weak* continuous. In fact, if it were so, then, according to the quoted theorem of zizler, $X^{*}$ would admit an equivalent dual locally uniformly rotund norm. In particular, $C([0, \Omega]) *$ would have such a norm, which is impossible, see [13].

of course, we would like to drop the assumption of smoothness in the above theorem. If it were possible, then this provide further support for the conjecture that each Asplund space admits an equivalent norm possessing some smoothness properties.

Remark. Recently Professor Ch. Stegall has kindly informed me that there exists another, more direct, way of showing that the mapping $D$ from the proof of Theorem 1 is of the first Baire class: According to a result of Bourgain [2] (Stegall has proved it also), a function $f: M \longrightarrow R$ defined on a complete metric space $M$ is of the first Baire class if and only if for every compact set $K \subset M$ the restriction $f / K$ has a point of continuity. By the Dugundji extension theorem we may replace $R$ by any Banach space. Let now $K \subset X$ be a compact set. Then, as our $D$ is norm-weak* continuous, $D(K)$ is weak* compact set. Now, since $X$ is Asplund, there are $k \in K$ and $x \in X$ such that $D k$ is strongly exposed by $x$. Thus, if $k_{n} \rightarrow k$, then $D k_{n}(x) \rightarrow D k(x)$ and hence $D k_{n} \rightarrow D k$ in norm. It means that $D / K$ is norm-norm continuous at $k$, 
and, by the quoted result, $D$ is of the first Baire class.

\section{References}

[1] D. Amir and J. Lindenstrauss, "The structure of weakly compact sets in Banach spaces", Ann. of Math. 88 (1968), 35-46.

[2] J. Bourgain, "Compact sets of first Baire class", BuZZ. Soc. Math. Belg. 29 (1977), 135-143.

[3] J. Diestel, "Geometry of Banach spaces - Selected topics", Lecture Notes in Math., 485, (Springer verlag 1975).

[4] J. Diestel and J.J. Uhl, Jr., "Vector measures", Mathematical Surveys - No. 15, (Amer. Math. Soc. Providence, Rhode Island 1977).

[5] M. Fabian, "On projectional resolution of identity on the duals of certain Banach spaces", Bull. Austral. Math. Soc. 35 (1987) 363-371.

[6] J.E. Jayne and C.A. Rogers, "Borel selectors for upper semicontinuous set-valued maps", Acta Math. 155 (1985), 41-79.

[7] R.W. Hansell, "On Borel mappings and Baire functions", Trans. Amer. Math. Soc. 194 (1974), 195-211.

[8] K. John and V. Zizler, "Projections in dual weakly compactly generated Banach spaces", Studia Math. 49 (1973), 41-50.

[9] K. John and V. Zizler, "A note on renorming of dual spaces", BuIZ. Acad. Pozon. Sci., Sér. Sci. Math. Astronom. Phys. 21 (1973), 47-50.

[10] K. John and V. Zizler, "Smoothness and its equivalents in weakly compactly generated Banach spaces", J. Funct. Anal. 15 (1974), 1-11.

[11] s. Mercourakis, "On weakly countably determined Banach spaces", Trans. Amer. Math. Soc. ( to appear).

[12] I. Namioka and R.R. Phelps, "Banach spaces which are Asplund spaces", Duke Math. J. 42 (1975), 735-750.

[13] M. Talagrand, "Renormages de quelques $C(K)$ ", Israel J. Math. 54 (1986), 327-339.

[14] L. Vałák, "On one generalization of weakly compactly generated Banach spaces", Studia Math. 70 (1981), 11-19. 
[15] v. Zizlex, "Locally uniformly rotund renorming and decompositions of Banach spaces", Bull. Austral. Math. Soc. 29 (1984), 259-265.

\section{Sibeliova 49}

16200 Prague 6

Czechoslovakia

Added in Proof: By combining Theorem 1 together with [12, Theorem 18] and [3, Theorem 4, page 152] we immediately get the following COROLLARY. Avery dispersed (that is, scattered) Gul'ko compact is Eberiein.

It should also be noted that it was Professor $K$. Musial who firstly suggested to us finding a Frechet differentiable norm in a weakly compactly generated Asplund space. 